FOLIA

Amazónica

Revista del Instituto de Investigaciones

de la Amazonía Peruana

\title{
EFECTO DE DIFERENTES CONCENTRACIONES DE ÁCIDO INDOLBUTÍRICO EN EL ENRAIZAMIENTO DE ESTAQUILLAS DE Annona muricata «GUANÁBANA» EN CÁMARA DE SUBIRRIGACIÓN
}

Víctor Reyger PÉREZ ORTIZ ${ }^{1}$, Nadia Masaya PANDURO TENAZOA ${ }^{1}$, José Gerardo SÁNCHEZ CHOY SÁNCHEZ ${ }^{1}$, Manuel Mario CHUYMA TOMAYLLA², Pablo Pedro VILLEGAS PANDURO².

${ }^{1}$ Universidad Nacional Intercultural de la Amazonía.

2 Universidad Nacional de Ucayali.

\section{RESUMEN}

La investigación tuvo como objetivo, determinar el efecto de diferentes concentraciones de Ácido Indolbutírico (AIB) en el enraizamiento de estaquillas de Annona muricata «Guanábana» en cámara de subirrigación, el cual se desarrolló en el vivero agroforestal de la Universidad Nacional Intercultural de la Amazonía (UNIA), ubicada en el km 0,63 de la carretera San José de Tushmo, distrito de Yarinacocha, provincia de Coronel Portillo, región Ucayali, Perú, entre los meses de Agosto a Octubre del 2017. Las estaquillas de guanábana fueron recolectadas, cortando las ramitas con tijera de podar, de 10-12 cm de longitud, y un diámetro de 0,5 cm, para luego cortar la mitad de las hojas con un bisturí. Las estaquillas se desinfectaron con fungicida, por 10 minutos y ventilándolos 10 minutos, luego fueron introducidas cerca de $1 \mathrm{~cm}$ de su base, en un envase conteniendo las diferentes soluciones de AIB, por un lapso de 3 segundos, y con la ayuda de un ventilador se secaron durante 1 minuto, con el fin de que el alcohol se evapore y pueda fijarse la hormona, para luego instalarlas dentro de la cámara de subirrigación de acuerdo a los tratamientos estudiados $(0$ ppm de AIB, 1000 ppm de AIB, 2000 ppm de AIB y 3000 ppm de AIB); concluyendo que, para el número de raíces, el tratamiento testigo (0 ppm de AIB) y el tratamiento con $2000 \mathrm{ppm}$ AIB, presentaron los mejores promedios (3,55 y 3,39 raíces respectivamente), no encontrándose diferencias significativas entre los tratamientos para la longitud de raíces, y para el porcentaje de estaquillas enraizadas, el tratamiento con 1000 ppm de AIB, presento el mejor promedio 
(41\%) y finalmente, para el porcentaje de sobrevivencia, el tratamiento con 2000 ppm de AIB presentó el mejor promedio (99,33\%).

PALABRAS CLAVE: Annona muricata, Ácido Indolbutírico, propagación asexual, cámara de subirrigación, estaquillas enraizadas, sobrevivencia.

\title{
EFFECT OF DIFFERENT CONCENTRATIONS OF INDOLBUTYRIC ACID IN THE ROOTING OF CUTTINGS OF Annona muricata «GUANÁBANA» IN SUBIRRIGATION CHAMBER
}

\begin{abstract}
The objective of the investigation was to determine the effect of different concentrations of Indolbutyric Acid (AIB) on the rooting of Annona muricata «Guanabana» cuttings in the sub-irrigation chamber, which was developed in the agroforestry vivarium of the Universidad Nacional Intercultural de la Amazonía (UNIA), located at km 0.63 of the San José de Tushmo road, Yarinacocha district, Coronel Portillo province, Ucayali region, Perú, between the months of August to October of 2017. Soursop cuttings were collected, cutting the twigs with pruning shears, with 10-12 cm in length, and a diameter of $0.5 \mathrm{~cm}$, and then cutting half of the leaves with a scalpel. The cuttings were disinfected with fungicide, for 10 minutes and ventilated for 10 minutes, then they were introduced about 1 $\mathrm{cm}$ from their base, in a container containing the different AIB solutions, by 3 seconds, and with the help of a fan were dried for 1 minute, so that the alcohol evaporates and the hormone can be fixed, and then installed inside the subirrigation chamber according to the treatments studied (0 ppm of AIB, 1000 ppm of AIB, $2000 \mathrm{ppm}$ of AIB and $3000 \mathrm{ppm}$ of AIB); concluding that, for the number of roots, the control treatment ( $0 \mathrm{ppm}$ of AIB) and the treatment with 2000 ppm AIB, presented the best averages ( 3.55 and 3.39 roots respectively), not finding significant differences between treatments for the length of roots, and for the percentage of rooted cuttings, the treatment with $1000 \mathrm{ppm}$ of AIB, presented the best average (41\%) and finally, for the percentage of survival, the treatment with $2000 \mathrm{ppm}$ of AIB presented the best average (99.33\%).

KEYWORDS: Annona muricata, Indolbutyric acid, asexual propagation, subirrigation chamber, rooted cuttings, survival.
\end{abstract}




\section{INTRODUCCIÓN}

Actualmente en el mundo existe una demanda creciente por el consumo de sabores exóticos (Pinto et al., 2005), siendo la guanábana una fruta exótica apreciada por su sabor agradable, aroma, pulpa sub-ácida y jugosa; lo que la convierte en un insumo potencial para producir puré, jugo, mermelada, jalea, barras dulces y postres (Abbo $e t$ al., 2006), además de su interesante composición nutricional (Ojeda et al., 2007).

Por otro lado en el área de la farmacologia, los tallos, hojas, semillas y frutos eran usados históricamente en medicina tradicional por pueblos indígenas de América del Sur debido a sus aportes benéficos como antitumorales y antiparasitarios (Solís et al., 2010), además de incrementar la leche de las madres después del parto (Baskar et al., 2007; Álvarez, 2007).

En el Brasil tradicionalmente las hojas de guanábana son usadas para el tratamiento de enfermedades hepáticas (Solís et al., 2010), supurativas, conjuntamente por su efecto antipirético (Badrie y Schauss, 2010) y antiinflamatorio (Gómez et al., 2009, Vieira de Sousa et al., 2010).

La propagación vegetativa o asexual se utiliza para producir una planta que posea el mismo genotipo que la planta madre (Hartmann y Kester, 1992). En ese sentido el uso de la propagación vegetativa es oportuna porque ofrece ventajas para generar plantaciones con mayor productividad, mejor calidad del producto; ganancia genética, al capturar tanto los componentes aditivos como no aditivos de la variación genética total; mayor homogeneidad en plantaciones y facilidad de manejo (Mesen, 2008).

Sin embargo, el desarrollo vegetal está influenciado, entre otros factores, por sustancias como los reguladores de crecimiento y las hormonas, dentro de ellas las auxinas, responsables de iniciar la división celular y la elongación, asi mismo aumenta el transporte de carbohidratos y cofactores foliares a la base de la estaca, para promover el desarrollo y formación de primordios (Haissig, 1974, citado por Núñez, 1997; Jordan y Casaretto, 2006).

Una forma predominante de auxinas en las plantas es el Ácido Indolacético (IAA), muy activo en bioensayos y presente comúnmente en concentraciones nanomolares. Otras formas naturales de auxinas son el Ácido 4-cloro-Indolacético (4-CIIAA), Ácido Fenilacético (PAA), Ácido Indolbutírico (IBA) y el Ácido Indol propiónico (Ludwig-Müller \& Cohen, 2002).

El Ácido Indolbutírico (AIB), es una auxina sintética químicamente similar al Acido Indolacético (AIA) la cual, ofrece muchas ventajas, ya que no se degrada fácilmente por la luz o microorganismos, es insoluble en agua, no es tóxico y permanece por más tiempo en el sitio de aplicación (Mesén, 1998).

Investigaciones realizadas por Orosco y Gonzales (2013) con Annona diversifolia Saff. en dos fenofases (crecimiento y reproducción), indicaron que el tiempo en el que permanecen los esquejes en el sustrato a partir de su siembra, no es un factor que determine el número de esquejes vivos y la tasa de sobrevivencia, sin embargo, la correlación encontrada entre la fase fisiológica de la planta madre con los esquejes vivos indica que es el factor más influyente.

Ademas de eso, Iglesias y Sánchez (1985) estudiaron el comportamiento de A. muricata L., A. reticulata, A. montana Mac y A. squamosa $\mathrm{L}$. cuyos mayores rendimientos fueron en injertos de parche en guanábano (82.5\%), empalme de costado en guanábano (47.5\%), parche en guanábana del Chocó (47.5 \%) y empalme de costado en anona colorada (35.0\%).

Con todo lo expuesto el objetivo de este trabajo fue evaluar el efecto de diferentes concentraciones 
de Ácido Indolbutírico (AIB) en el enraizamiento de estaquillas de Annona muricata «Guanábana» en el vivero de la UNIA.

\section{MATERIALES Y MÉTODOS}

\section{UBICACIÓN DEL EXPERIMENTO}

El experimento se llevó a cabo en el Vivero Agroforestal de la Universidad Nacional Intercultural de la Amazonía, ubicada en el km 0,63 de la carretera San José de Tushmo, distrito de Yarinacocha, provincia de Coronel Portillo, región Ucayali, Perú.

Las estaquillas de Anonna muricata fueron recolectadas de una plantación ubicado en el fundo «Los Gemelos», ubicado en la margen izquierdo de la carretera Federico Basadre km 26, interior $2 \mathrm{~km}$.

\section{PROCEDIMIENTO}

\section{A. Recolección del material vegetativo}

La recolección se realizó por la mañana (6 a.m.), seleccionando plantas madres con frutos de buen tamaño y estado sanitario adecuado. Se procedió a identificar la parte media y apical, luego con ayuda de bisturí fueron cortadas las hojas por la mitad, para preparar estacas de 10-12 $\mathrm{cm}$ de longitud y $0,5 \mathrm{~cm}$ de diámetro. Finalmente las estacas fueron envueltas en papel periódico húmedo y trasladadas en hieleras de tecnopor para evitar que se deshidraten.

\section{B. Acondicionamiento en cámara de subirrigación}

Fue utilizada una cámara de subirrigación elaborada con madera pre-dimensionada, de $1 \mathrm{~m}$ de ancho por $1 \mathrm{~m}$ de largo y $1 \mathrm{~m}$ de altura, forrada con mica translucida de polietileno, en cuyo interior se colocaron varias capas sucesivas de piedra, grava, arena y aproximadamente 30 litros de agua, a fin de irrigar las estaquillas y retener la humedad necesaria entre las paredes de la cámara herméticamente cerrada.

\section{Preparación y aplicación de las dosis hormonales}

Se prepararon concentraciones de AIB: 1000 ppm, 2000 ppm y 3000 ppm en una solución de alcohol de 96\%. Las estacas se desinfectaron con una solución fungicida y ventilaron por 10 minutos para ser introducidas cerca de 1 $\mathrm{cm}$ de su base, inmediatamente se remojaron durante 3 segundos en envases conteniendo las soluciones hormonales, para luego instalar las estacas dentro de la cámara de subirrigación según la ubicación de los tratamientos.

\section{Monitoreo}

La evaluación se realizó al finalizar los dos meses (60 días), evaluando la longitud y número de raíces, porcentaje de enraizamiento y porcentaje de sobrevivencia.

\section{TRATAMIENTO ESTADÍSTICO}

Se utilizó un diseño completamente al azar (DCA) con cuatro tratamientos y tres repeticiones, haciendo un total de 12 unidades experimentales, y cada unidad experimental contó con 12 estacas, usando un total de 144 estacas.

Se aplicó el análisis de varianza (ANOVA) y la prueba de comparación de las medias de Tukey $(\mathrm{alfa}=0,05)$, con el fin de probar si existen diferencias significativas entre los tratamientos de AIB. Para el análisis estadístico, se utilizó el programa informático SPSS versión 21. 


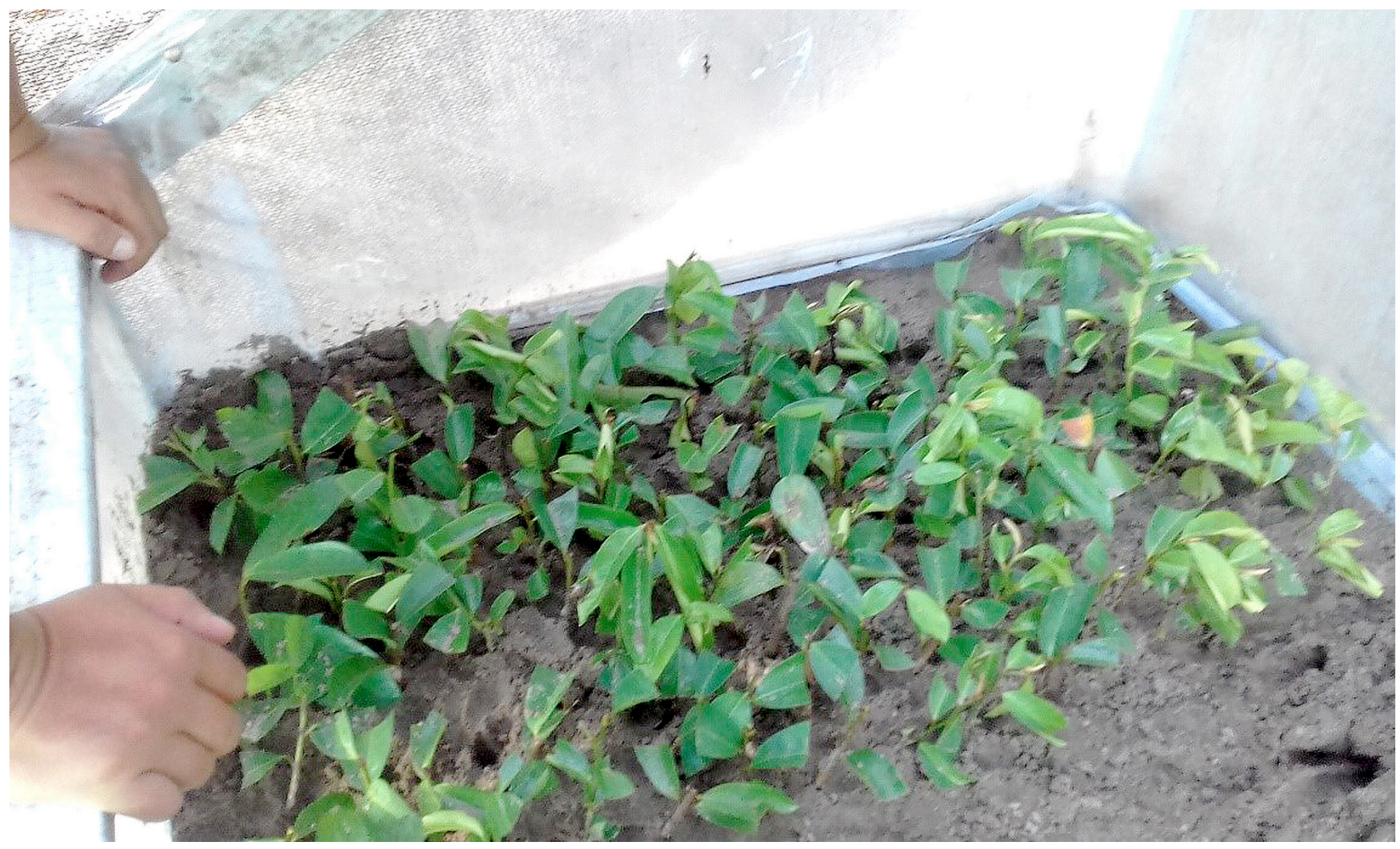

Figura 1. Estaquillas de guanábana (Annona muricata L.) tratadas con diferentes concentraciones de AlB en el interior de la cámara de subirrigación.
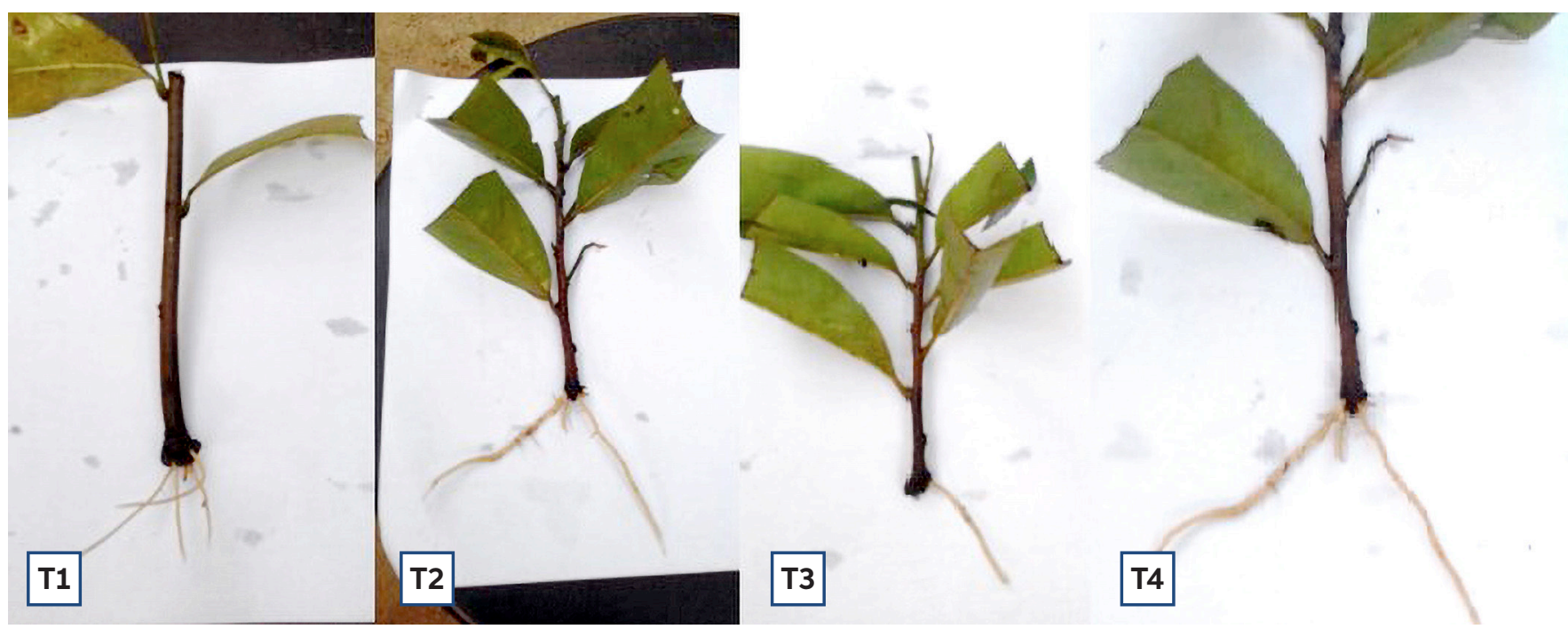

Figura 2. Respuesta de las estaquillas de guanábana (Annona muricata L.) a la aplicación del AIB. 


\section{RESULTADOS Y DISCUSIONES}

NÚMERO DE RAÍCES, LONGITUD DE RAÍCES Y PORCENTAJE DE ESTAQUILLAS ENRAIZADAS

La tabla 1, muestra la prueba de promedios de Tukey, para el número de raíces, longitud de raíces y porcentaje de estaquillas enraizadas.

Tabla 1. Prueba de promedios de Tukey, para el número de raíces, longitud de raíces y porcentaje de estaquillas enraizadas de guanábana a los 60 días de instalado.

\begin{tabular}{ccccc}
\hline Tratamientos & Descripción & No de raíces & $\begin{array}{c}\text { Longitud de } \\
\text { raíces (cm) }\end{array}$ & $\begin{array}{c}\text { Porcentaje } \\
\text { de estaquillas } \\
\text { enraizadas (\%) }\end{array}$ \\
\hline 1 & 0 ppm AlB & $3,58 \mathrm{a}$ & $3,99 \mathrm{a}$ & $39,00 \mathrm{a}$ \\
2 & 1000 ppm AIB & $3,36 \mathrm{a}$ & $3,29 \mathrm{a}$ & $41,00 \mathrm{a}$ \\
3 & 2000 ppm AIB & $3,40 \mathrm{a}$ & $3,15 \mathrm{a}$ & $21,00 \mathrm{~b}$ \\
4 & 3000 ppm AIB & $2,08 \mathrm{~b}$ & $4,76 \mathrm{a}$ & $20,66 \mathrm{~b}$ \\
\hline
\end{tabular}

meristemo apical de la raíz y contribuyen con el patrón de generación de nuevas raíces laterales, siendo las auxinas las que juegan un papel crucial (Jovanovic et al., 2008; Ljung et al., 2005). La aplicación de auxinas acelera la iniciación radical, aumenta el número de estacas enraizadas, incrementa el número y la calidad de las raíces, además de proporcionar una mayor uniformidad en el crecimiento y desarrollo de las raíces (Bacarín et al., 1994), sin embargo, se observó que las estaquillas sin aplicación de AIB, generaron raíces, lo cual demuestra que la guanábana no requieren estímulos a través de la aplicación de AIB.

Una característica in-
Para el número de raíces, la prueba de promedios de Tukey muestra que diferencias significativas, en los cuales, los tratamientos con 0 ppm de AIB, 1000 ppm de AIB y 2000 ppm de AIB, mostraron los mejores promedios de número de raíces, observándose diferencias significativas con respecto al tratamiento con 3000 ppm de AIB. En cuanto a la longitud de raíces, la prueba de promedios de Tukey no muestra diferencias significativas entre los tratamientos estudiados. Asimismo, para el porcentaje de estaquillas enraizadas, la prueba de promedios de Tukey muestra que los tratamientos con 0 ppm de AIB y 1000 ppm de AIB presentaron los mejores porcentaje de estaquillas enraizadas (39\% y $41 \%$ respectivamente),los cualesmostrarondiferencias significativas con respecto a los tratamientos con 2000 ppm (21\%) y el tratamiento con 3000 ppm de AIB $(20,66 \%)$.

El crecimiento de la raíz es regulado por señales endógenas que mantienen la actividad del dispensable para el enraizado de estacas en especies leñosas es el uso de tejido juvenil (Iglesias et al., 1996), por lo que es común utilizar plantas jóvenes o rebrotes juveniles de plantas de mayor edad (Chaturvedi et al., 1996; Palanisamy y Kumar, 1997). Pero aún con rebrotes juveniles la capacidad de enraizado de las estacas es afectada por otros factores fisiológicos y ambientales. Entre los primeros se incluye la concentración endógena de fitohormonas, las reservas de carbohidratos y el grado de lignificación del tallo (Veierskov, 1988; Mateo et al., 2000), estas afirmaciones explican los resultados obtenidos en el presente estudio, en el cual las estaquillas juveniles de guanábana utilizadas sin aplicación de AIB, generaron formación de raíces de manera similar a las estacas que sí tuvieron aplicación de AIB.

\section{PORCENTAJE DE SOBREVIVENCIA}

La tabla 2, muestra la prueba de promedios de Tukey, para el porcentaje de sobrevivencia. 
Tabla 2. Prueba de promedios de Tukey, para el porcentaje de sobrevivencia de estaquillas de guanábana a los 60 días de instalado.

\begin{tabular}{ccc}
\hline Tratamientos & Descripción & \% Sobrevivencia \\
\hline 1 & 0 ppm AIB & $84,00 \mathrm{~b}$ \\
2 & 1000 ppm AIB & $73,00 \mathrm{~d}$ \\
3 & 2000 ppm AIB & $99,33 \mathrm{a}$ \\
4 & 3000 ppm AIB & $78,00 \mathrm{c}$ \\
\hline
\end{tabular}

Para el porcentaje de sobrevivencia, la prueba de promedios de Tukey demuestra que el tratamiento con 2000 ppm de AIB presentó el mayor porcentaje de sobrevivencia de estaquillas, el cual mostró diferencias significativas con respecto al tratamiento con 0 ppm de AIB, el cual mostró diferencias significativas con respecto al tratamiento con 3000 ppm de AIB, el mismo que mostró diferencias significativas con respecto al tratamiento con 1000 ppm de AIB.

El AIB tiene una débil actividad auxínica en general pero una excelente acción rizógena (Boutherin y Bron, 1994). Sin embargo, el AIB es probablemente el mejor enraizador para uso masivo, debido a que no es tóxico para las plantas en una amplia gama de concentraciones y es efectivo para estimular el enraizamiento de un gran número de especies de plantas (Hartmann y Kester, 1999). La mayoría de las especies forestales enraízan bien con dosis de $0,2 \%$ a $0.3 \%$ de AIB, aunque algunas pueden requerir dosis mayores o menores (Soudre et al., 2008; Mesen, 1998).

\section{CONCLUSIONES}

1. Las estaquillas sin aplicación de AIB, enraizaron igual que las estacas con aplicación de AIB, siendo la aplicación con 0 ppm AIB y 1000 ppm de AIB los que presentaron los mejores promedios de estacas enraizadas (39 \% y 41 $\%$ respectivamente) y número de raíces no encontrándose diferencias significativas para la longitud de raíces.

2. Para el porcentaje de sobrevivencia, el tratamiento con 2000 ppm de AIB presentó el mejor promedio $(99,33 \%)$.

\section{REFERENCIAS BIBLIOGRÁFICAS}

Abbo, E.S.; Olurin, T.; Odeyemi, G. 2006. Studies on the storage stability of soursop (Annona muricata L.) juice. Afr J Biotechnol 5: 108-112.

Álvarez, P. 2007. Decisiones en reacciones adversas a medicamentos, intoxicaciones $\mathrm{y}$ respuestas inesperadas de productos naturales como problemas de salud pública. Rev Perú Med Exp Salud Publica 24: 405-426.

Bacarín, M.; Benincasa, M.; Andrade, V.; Fereira, E.F. 1994. Enraizamiento de estacas aéreas de goiabeira (Psidium guajava L.): Efeito do ácido indolbutírico sobre a iniciacao radicular. Revista Científica, Sao Paulo 22: 71-79.

Badrie, N.; Schauss A. 2010. Soursop (Annona muricata L.): composition, nutritional value, medicinal uses, and toxicology. 621-643. En Bioactive foods in promoting health: fruits and vegetables. Watson RR, Preedy VR [Eds]. Elsevier Inc. Oxford, UK.

Baskar, R.; Rajeswari, V.; Sathish-Kumar, T. 2007. In vitro antioxidant studies in leaves of Annona species. Indian J Exp Biol 45: 480-485.

Boutherin, D.; Bron, G. 1989. Multiplicación de plantas hortícolas. Instituto Agrícola y Hortícola Anger. 225 pp.

Chaturvedi, O.P.; Jha, A.N.; Das, D.K. 1996. Vegetative propagation of Acacia auriculiformis by stem cuttings. Forest, Farm, and Community Tree Research Reports. Vol. 1. Department of Forestry, Rajendra Agricultural University. Bihar, India. pp: 41-44. 
Correa Gordillo, J.; Ortiz, D.; Larrahondo, J. E.; Sánchez Mejía, M.; Pachón, H. 2012. Actividad antioxidante en guanábana (Annona muricata L.): una revisión bibliográfica Boletín Latinoamericano y del Caribe de Plantas Medicinales y Aromáticas, vol. 11, núm. 2, 2012, 111-126. Universidad de Santiago de Chile. Santiago, Chile. Disponible en: http://www. redalyc.org/articulo.oa?id=85622734002

Gómez, H.; Germosen Robineau, L.; Nossin, E. 2009. Estudio etnofarmacológico de las plantas medicinales usadas en el Caribe colombiano. En Reyes G. Diálogo de saberes: plantas medicinales, salud y cosmovisiones. Universidad Nacional de Colombia, Sede Amazonia. ARFO Editores e Impresos Ltda. Bogotá, Colombia.

Hartmann, H.T; Kester, D.; Davies, F.T. 1992. Plant propagation. Principles and practices. Fifth.

Hartmann, H. T.; Kester, D.E. 1999. Propagación de Plantas: Principios y Prácticas. Traducción al Español por Antonio Marino. 7a reimpresión, Ed. CECSA. México D. F., México. 219-363.

Iglesias, G.L.; Alarcón, M.; Prieto, J.A. 1996. La propagación vegetativa de plantas forestales. Ciencia For. Méx. 21: 15-41.

Iglesias A, I., Luis A.Sánchez,L.A. 1985. Propagación del "Guanabano", Annona muricata L., por medio de injerto sobre diferentes patrones de Anonaceas. Acta Agron. Vol. 35 (3) 53-58.

Jovanovic, M.; Lefebvre, V.; Laporte, P.; González Rizzo, S.; Lelandais Brière, C.; Frugier, F; Hartmann, C.; Crespi, M. 2008. How the environment regulates root architecture in dicots. Advances in Botanical Research 46: 3574.

Jordán, M. y Casaretto, J. 2006. Fisiología Vegetal (F.A. Squeo \& L. Cardemil, eds.) Ediciones Universidad de La Serena, La Serena, Chile (2006). Capítulo XV Hormonas y Reguladores del Crecimiento: Auxinas, Giberelinas y Citocininas. Disponible en: http://www.exa. unne.edu.ar/biologia/fisiologia.vegetal/ Auxinasgiberelinasycitocininas.pdf

Ljung, K.; Hull, A.K.; Celenza, J.; Yamada, M.; Estelle, M.; Normanly, J.; Sandberg, G. 2005. Sites and regulation of auxin biosynthesis in Arabidopsis roots. Plant Cell 17: 1090-1104.

Ludwig Müller, J \& Cohen, J.D. 2002. Identification and quantification of three active auxins in different tissues of Tropaeolum majus. Physiologia Plantarum 115: 320-329.

Mateo, S.; Vargas, H.; López, P.; Jasso, M. 2000. Enraizado de estacas juveniles de cinco especies de coníferas ornamentales. Ciencia For. México. 23: 29-39.

Mesén, F. 2008. Curso: Bases Técnicas Para la Propagación Vegetativa de Árboles Tropicales Mediante Enraizamiento de Estaquillas. Pucallpa, Perú.

Mesén, F. 1998. Enraizamiento de estacas juveniles de especies forestales: Uso de Propagadores de sub-Irrigación. Centro Agronómico Tropical de Investigación y Enseñanza - CATIE. Turrialba, Costa Rica. 33 pp.

Nuñes, Y. 1997. Propagación vegetativa del Cristóbal (Platymiscium pinnatum, Benth); pilon (Hyeromina alchorneoides, Allemo) y surá (Terminalia oblonga, Ruiz \& Pavon) mediante el enraizamiento de estacas juveniles Tesis Mg. Sc. CATIE. Turrialba, Costa Rica. 172 pp.

Ojeda, G.; Coronado, J.; Nava, R.; Sulbarán, B.; Araujo, D.; Cabrera, L. 2007. Caracterización fisicoquímica de la pulpa de la guanábana (Annona Muricata) cultivada en el Occidente de Venezuela. Bol Centro Invest Biol 41 (2): 151-160.

Palanisamy, K.; Kumar, P. 1997. Effect of position, size of cuttings and environmental factors on adventitious rooting in Neem (Azadirachta indica). For. Ecol. Manag. 98: 277-280.

Orozco-Castillo, J.A., González-Esquinca, A.R. 2013. Evaluación de la propagación asexual por esquejes en plantas juveniles de Annona 
diversifolia Saff. (Annonaceae). Revista de Ciencias de la Unicach Lacandonia 7, (7) 1: 1518, junio de 2013.

Pinto, A.C.; Cordeiro, M.C.; De Andrade, S.R.; Ferreira, F.R.; Filgueiras, H.A.; Alves, R.E.; Kinpara, D.I. 2005. Annona species. University of İnternational Southampton, Centre for Underutilised Crops. Southampton, UK.

Solís Fuentes, J.A.; Amador, C.; Hernández, M.R.; Duran, M.C. 2010. Caracterización fisicoquímica y comportamiento térmico del aceite de "almendra" de guanábana (Annona muricata, L). Grasas y Aceites 61: 58-66.

Soudre, M.; Meses, F.; Del Castillo, D.; Guerra, H. 2008. Memoria del curso internacional «Bases técnicas para la propagación vegetativa de árboles tropicales mediante enraizamiento de estaquillas» IIAP, Pucallpa. Perú. 100 pp.

Veierskov, B. 1988. Relations between carbohydrate and adventitious root formation. In: Adventitious Root Formation in Cuttings, T D Davis, B E Haissig, N Sankhla (eds). Dioscorides Press. Portland, Oregon, USA. 70-78.

Vieira De Sousa, O., Vieira, G.D.V.; Pinho, J.J.; Yamamoto, C.H.; Alves, M.S. 2010. Antinociceptive and anti-inflammatory activities of the etanol extract of Annona muricata L. leaves in animal models. Int J Mol Sci.11: 2067-2078.

Recibido: 3 de enero de 2019 Aceptado para publicación: 15 de mayo de 2019

Esta obra está bajo una Licencia Creative Commons Atribución-NoComercial-SinDerivar 4.0 Internacional. 